\title{
DEL EQUILIBRIO GENERAL COMPETITIVO A LA TEORÍA KEYNESIANA
}

\author{
Alain IzE \\ El Colegio de México
}

\section{INTRODUCCIón}

LA TEORÍA KEYNESIANA ha sido tradicionalmente presentada en forma aislada, sin relación con el cuerpo principal de la teoría de los precios y con las orientaciones microeconómicas usuales. Como consecuencia, la economía keynesiana se ha encerrado dentro del marco rígido del modelo post-keynesiano, con lo cual se limita así de manera drástica su desarrollo.

En los últimos dos decenios ha surgido una importante corriente de reacción, orientada hacia los elementos de transición de la microeconomía a la teoría keynesiana. Algunas de las contribuciones más importantes en ese sentido han sido las de Patinkin (1956), Clower (1965), Leijonhufvud (1968), Phelps (1970), Barro y Grossman (1971) y Malinvaud (1977), en las cuales se hace énfasis de manera principal en el estudio de las decisiones de intercambio y de producción de los agentes dentro de un contexto de desequilibrio y de información imperfecta.

Este ensayo intenta presentar dentro de la misma corriente una visión sintética de la transición de un modelo microeconómico de equilibrio general competitivo a un modelo macroeconómico keynesiano. Nuestro objetivo será demostrar que un análisis minucioso de esta transición rinde varios frutos importantes. Primero porque permite reubicar a la teoría keynesiana en relación con la microeconomía tradicional y asegura así cierta progresión del pensamiento dentro del mismo marco de referencia. Segundo, porque el concepto de equilibrio general es un punto de referencia indispensable para entender los mecanismos y la dinámica de un sistema en desequilibrio. Por último, porque la teoría del equilibrio general competitivo ofrece un marco general de discusión que trasciende las limitantes de sus hipótesis de competencia perfecta y de equilibrio constante.

En la primera parte de este trabajo se sintetizan los rasgos más relevantes de la teoría del equilibrio general competitivo y se deducen de esta presentación los modelos clásicos y neoclásicos. En la segunda parte, se describe la transición al modelo keynesiano y en la tercera se intenta señalar las direcciones hacia las cuales es necesario desarrollar este modelo. En la última parte se esbozan algunas razones que justifican la 
relevancia de 1a teoría del equilibrio general como punto de partida para una exposición de la economía keynesiana.

\section{EL EQUILIBRIO GENERAL COMPETITIVO}

\section{Formulación ${ }^{1}$}

Se considera una economía formada por $n$ consumidores y $m$ empresas que intercambian bienes y servicios a través del tiempo. Se definen precios de valor presente para todos los bienes y todas las fechas: $P_{i t}$ expresa así el precio del bien $i$ pagado ahora para que sea intercambiado en el período $t$. Se definen también tasas de interés propias para cada bien y cada par de fechas: $S_{i}(0, t)$ es la tasa de interés propia del bien $i$ entre el período $t$ y el período cero. Esta tasa se define como: ${ }^{2}$

$$
S_{i}(0, t)=\frac{P_{i 0}}{P_{i t}}=1
$$

Los consumidores disponen en cada período de acervos iniciales de bienes y servicios, por ejemplo el vector $\bar{x}_{h}$ para el consumidor $h$. Intercambian cantidades $x_{h}$ (positivas para ventas, negativas para compras) de tal forma que maximizan la utilidad derivada del consumo a través del tiempo pero se respeta la restricción presupuestal de que el valor de sus compras no rebase el valor de sus ventas. El problema del consumidor entonces es:

$$
\begin{aligned}
& \operatorname{Max} U\left(\bar{x}_{h}-x_{h}\right) \\
& x_{h} \\
& \text { dado que: } P x_{h} \geqslant 0 \quad \text { para } h=1,2, \ldots n
\end{aligned}
$$

donde $P$ representa la matriz intertemporal de precios. Al resolver este problema de optimización se puede deducir para cada consumidor un vector de ofertas netas (o "excesos" de oferta) que se expresan en función de los parámetros del problema, o sea de sus preferencias (implícitas), de sus acervos y de los precios: $x_{h}\left(P, \bar{x}_{h}\right)$.

Por su parte las empresas maximizan sus ganancias teniendo en cuenta sus restricciones de producción. Se establece la hipótesis de com-

1 Para una formulación rigurosa y completa véase Arrow y Hahn (1971).

2 A partir de esta definición es fácil deducir que la condición para que todas las tasas de interés propias entre dos períodos sean iguales entre sí es que los precios relativos de todos los bienes sean los mismos en los dos períodos. Por otra parte, para que las tasas de interés entre dos pares de períodos consecutivos sean iguales es necesario que los precios suban o bajen en forma constante a través del tiempo. 
petencia perfecta, o sea que las empresas aceptan los precios de mercado y no tienen individualmente control o impacto sobre ellos. Si $Y_{f}$ es el vector de producción de la empresa $f$ (con insumos negativos y producción positiva) y si $F_{f}$ corresponde al conjunto de sus posibilidades tecnológicas de producción, el comportamiento de la empresa se puede expresar entonces como:

$$
\begin{aligned}
& \operatorname{Max} P Y_{f} \\
& Y_{f} \\
& \text { dado que: } Y_{f} \in F_{f} \quad \text { para } f=1,2, \ldots m
\end{aligned}
$$

De esta maximización se deriva un vector de ofertas netas que es función de los precios y de las restricciones tecnológicas (implícitas): $Y_{f}(P)$.

Al agregar todas las ofertas netas individuales se deriva por último un vector agregado de ofertas netas que es función de los precios y de la distribución de acervos iniciales (e implícitamente de las preferencias y de las restricciones tecnológicas):

$$
Z(P, \bar{x})=\sum_{h=1}^{n} x_{h}\left(P ; \bar{x}_{h}\right)+\sum_{f=1}^{M} Y_{f}(P)
$$

Estas ofertas agregadas se expresan únicamente en función de los precios de mercado (y también de los acervos) pero no en función de cantidades intercambiables ya que corresponden a los deseos de intercambio de los agentes en la ausencia de toda restricción sobre cantidades que se intercambian efectivamente en todos los mercados. En la terminología de Clower son ofertas "nocionales" o sea ofertas que existirían si todos los agentes estuvieran seguros de que puedan intercambiar tanto como quisieran. Veremos después que esta distinción es un elemento crucial de la transición al modelo keynesiano.

Por otra parte, es importante notar que según la definición de las tasas de interés propias - relación (1) - , los precios de bienes intercambiables en el futuro se pueden expresar en función de los precios de los bienes intercambiados hoy (precios corrientes) y de las tasas de interés. Las ofertas y demandas agregadas se pueden entonces expresar en forma alternativa en función de los precios de hoy, de las tasas de interés y de los acervos:

$$
Z\left(P_{o}, S, \bar{x}\right)
$$

\section{Existencia, eficiencia y unicidad del equilibrio}

El teorema principal de la teoría del equilibrio general es que bajo condiciones de convexidad de los conjuntos de preferencia y de los conjuntos productivos existe por lo menos un sistema de precios que permite eliminar todos los excesos de demanda y que sea tal que si existe un exceso 
de oferta en algún mercado, el precio correspondiente deba ser nulo. ${ }^{3}$ Si se deja de lado el caso de los bienes gratuitos, este resultado significa que es posible encontrar en un sistema descentralizado, en el cual cada agente maximiza su interés individual, por lo menos un sistema de precios que permita equilibrar todos los mercados. Si la economía está en ese punto no existe entonces desempleo involuntario ni inflación por exceso de demanda. Todos los agentes consumen, trabajan y producen tanto como desean ${ }^{4} \mathrm{y}$ todos los planes individuales son consistentes.

Se puede además demostrar, bajo condiciones de convexidad estricta, que existe sólo un punto de equilibrio. Por último, se demuestra que ese punto de equilibrio de mercados es eficiente en el sentido de Pareto: no se puede mejorar la posición de ningún agente sin empeorar la de otros.

\section{Búsqueda del equilibrio}

La existencia de un vector de precios de equilibrio no implica que la economía pueda en cada instante y bajo condiciones cambiantes seleccionar el vector apropiado. Walras, Marshall y Edgeworth han propuesto mecanismos de selección de un vector de precios de equilibrio.

El mecanismo walrasiano es el bien conocido "tatonnement". Existe un coordinador central que se encarga de fijar precios. Emite un vector inicial de precios y los agentes expresan sus ofertas y demandas a esos precios. Suma entonces ofertas y demandas en cada mercado e incrementa precios de los bienes con exceso de demanda, y reduce los precios de los bienes con exceso de oferta. Los agentes expresan de nuevo sus ofertas y demandas a estos precios, etc. Se demuestra que bajo ciertas condiciones de sustituibilidad de los bienes el proceso converge hacia el equilibrio.

El "mercado de pescados" de Marshall funciona con base en ajustes por cantidades en vez de precios. El domingo, día de mercado, las cantidades de pescado a la venta son fijas y se tienen que vender en ese día ya que el pescado no se puede conservar. El precio de venta (o precio de "demanda") se fija entonces de tal forma que permita igualar la demanda a la oferta de pescado. Con base en ese precio de demanda, los pescadores estiman un precio probable de venta el domingo siguiente (precio de oferta) y se dedican a pescar la cantidad de pescado que iguale el costo marginal de producción con el precio de oferta. Al cabo de suficientes semanas y bajo ciertas condiciones sobre las funciones de oferta y demanda se debería converger hacia un equilibrio en el cual el precio de demanda iguale el precio de oferta. ${ }^{5}$ Conforme a la tradición marshalliana de equilibrios parciales, este proceso se limita al caso de un solo mercado.

3 Este sería el caso por ejemplo del aire que respiramos (por lo menos fuera de la ciudad de México).

4 Pero obviamente bajo las condiciones expresadas antes de la observación del equilibrio presupuestal y de pertenencia al conjunto productivo.

5 Se ha argumentado recientemente que el proceso de ajuste marshalliano es 
El proceso concebido por Edgeworth se basa en negociaciones directas entre todos los agentes. La única condición impuesta es que los agentes negocien siempre acuerdos que beneficien a ambas partes. Se demuestra que cuando el número de agentes crece suficientemente y en la ausencia de coaliciones, el punto de equilibrio de una economía perfectamente competitiva se alcanza eventualmente mediante este proceso de negociaciones y "renegociaciones", punto en el cual ya no se puede realizar ninguna transacción adicional entre dos agentes que sea provechosa para ambos. ${ }^{\mathrm{B}}$

Estos modelos de búsqueda de un equilibrio son claramente poco realistas pero son interesantes porque permiten tener cierta idea de la mag. nitud del intercambio de información que se necesita para alcanzar el equilibrio, magnitud que es obviamente gigantesca. Dadas las limitaciones que puedan existir en una economía real sobre capacidades de procesamiento e intercambio de información y dadas las alteraciones constantes que tienden a producirse en los parámetros de intercambio, es entonces bastante claro reconocer que la economía pueda encontrarse con frecuencia fuera de equilibrio.

\section{Extensiones}

La teoría del equilibrio general competitivo en su formulación más estricta considera que todos los mercados existen para todos los bienes y para todas las fechas y que no hay dinero en la economía. Ambas restricciones pueden hasta cierto punto ser eliminadas del modelo.

En ausencia de mercados futuros, los precios de mercado pueden ser sustituidos por expectativas de precios y es claro que no se alterara nada el estado de equilibrio general si las expectativas de todos los agentes corresponden a los precios que hubieran existido en presencia de un conjunto completo de mercados. El estado de equilibrio general se caracteriza entonces por un equilibrio de demandas y ofertas en todos los mercados presentes y por la existencia de un conjunto de expectativas "correctas" o "racionales". Es importante observar que la condición para que esas expectativas correspondan a los precios que se hubieran formado si los mercados hubiesen existido, es que no haya habido especulación sobre comportamientos mutuos; o sea que ningún agente forme sus expectativas en una situación de especulación sobre el comportamiento de otros agentes, los cuales no deberían tampoco especular sobre los demás, etcétera... ${ }^{7}$ Como se verá después, la inclusión de expectativas especu-

más realista que el proceso walrasiano y por lo tanto más interesante. Véase Leijonhufvud (1974).

6 Para una buena discusión de las ventajas comparativas de los procesos walrasianos y edgeworthianos dentro de un contexto keynesiano, véase el artículo de Weintraub (1977).

7 Sobre estos conceptos véase Radner (1972) y Heller y Starr (1976). 
lativas aporta un elemento de interdependencia directa entre agentes que no permite asegurar la eficiencia de un equilibrio así establecido.

En el caso en que existe un equilibrio de todos los mercados presentes pero que las expectativas no sean correctas se puede simplemente hablar de equilibrio "temporal". Se demuestra que si las expectativas de precios futuros no se ven afectadas de manera muy brusca por un cambio en las condiciones actuales de intercambio, existe en general un vector de precios corrientes que permita asegurar un estado de equilibrio temporal. ${ }^{8}$

El dinero puede ser introducido, aunque hasta ahora en forma un poco artificial, en el modelo de equilibrio general y se llega a la conclusión que las ofertas y demandas son homogéneas de grado cero en los precios' y en la cantidad de dinero, ${ }^{9}$ o sea que una alteración proporcional de los precios y de la cantidad de dinero no alteran el estado de equilibrio. ${ }^{10}$ En la medida que la economía permanezca en equilibrio, el dinero es entonces un velo que no altera las condiciones de intercambio pero que permite fijar el nivel de precios. ${ }^{11}$

\section{Modelos agregados derivados}

Los modelos agregados tanto neoclásicos como clásicos se sustentan en el concepto de una economía que permanece constantemente en equilibrio.

El modelo neoclásico en su versión más sencilla es en esencia un modelo de equilibrio general que se limita a tres mercados, los de trabajo, bienes y dinero. Los bienes de inversión y los bienes de consumo son agregados dentro de un solo sector y se establece la hipótesis implícita de que los precios relativos futuros de los bienes y del trabajo son constantes a través del tiempo, lo que permite definir una tasa de interés única. Se dejan de lado efectos de distribución, tanto de acervos monetarios como de otros acervos y se examina un equilibrio de corto plazo en los mercados actuales: el acervo de capital es por lo tanto considerado como fijo dentro del ámbito del modelo. Dadas las homogeneidades de grado cero en los precios y en la cantidad de dinero, las ecuaciones

8 Sobre el concepto de equilibrio temporal véase Green (1973) y Grandmont (1976).

9 La referencia básica en esta área es Patinkin (1956) pero ha habido últimamente más trabajos en esta dirección. Véase en particular Grandmont y Younes (1976).

10 Sin embargo, esto no significa que al duplicar la cantidad de dinero se dupliquen automáticamente los precios; esta conclusión es válida sólo si se establece la hipótesis de que el sistema permanece en equilibrio y que ese equilibrio es único.

11 Es interesante apreciar que un estado de inflación permanente debido a un incremento permanente de la masa monetaria es perfectamente compatible con un estado de equilibrio general; o sea que el estado de equilibrio general no implica estabilidad de precios por necesidad. 
de equilibrio se pueden entonces escribir en la siguiente forma walrasiana;

en el mercado de trabajo: $L^{\circ}\left(\frac{w}{P}, r, \frac{M}{P}\right)=L^{d}\left(\frac{w}{P}, r, \frac{M}{P}\right)$

en el mercado de bienes: $Y^{o}\left(\frac{w}{P}, r, \frac{M}{P}\right)=Y^{d}\left(\frac{w}{P}, r, \frac{M}{P}\right)$

en el mercado de dinero: $M^{o}=M^{d}\left(\frac{w}{P}, r, \frac{M}{P}\right)$

Esto define un sistema de tres ecuaciones con tres variables, $w, p$ y $r$. El equilibrio queda por lo tanto bien definido. ${ }^{12}$

El modelo clásico parte de la misma filosofía de equilibrio pero está moldeado dentro de un marco de análisis parcial marshalliano. Las empresas deciden en forma separada cuánta mano de obra contratar para la producción de hoy y cuánta inversión realizar en previsión de la producción de mañana. Los consumidores deciden también en forma separada cuánto trabajo ofrecer hoy y cómo dividir su consumo entre hoy y mañana. Por otra parte, el acervo de dinero es eliminado del modelo. En el mercado de trabajo, la condición de equilibrio se reduce entonces a:

$$
L^{o}\left(\frac{w}{P}\right)=L^{d}\left(\frac{w}{P}\right)
$$

Este equilibrio permite determinar en forma aislada el nivel de empleo y el salario real. El equilibrio en el mercado de bienes se traduce en la ecuación:

$$
Y^{o}\left(\frac{w}{p}\right)=C(r, Y)+I(r)
$$

la función consumo se expresa en forma marshalliana en función de la tasa de interés y de cierto nivel de ingreso tomado como parámetro. Esta ecuación permite determinar la tasa de interés $(w / P$ ya fijada por el mercado de trabajo), y el nivel de ingreso. La ecuación de la teoría cuantitativa cierra el modelo;

en su forma fisheriana: $M v=P Y$

o marshalliana: $M=K P Y$

La cantidad de dinero fija por lo tanto el nivel de precios.

12 Dada la unicidad del equilibrio y bajo la hipótesis de que el sistema permanece siempre en equilibrio, es claro que un cambio en la masa monetaria no altera la naturaleza del equilibrio sino que se repercute únicamente en el nivel de precios. Existe una dicotomía entre el lado real y el lado monetario. 


\section{LA TRANSICIÓN AL MODELO KEYNESIANO}

\section{Equilibrio bajo restricciones}

El elemento medular de la transición del modelo de equilibrio general competitivo al modelo keynesiano con seguridad es la distinción entre un equilibrio con y sin restricciones, o bien, conforme a la terminología de Clower, la distinción entre demandas nocionales y demandas efectivas. En el modelo walrasiano los agentes expresan sus ofertas y demandas bajo la convicción de que podrán efectivamente comprar y vender tanto como ellos desearan. Pero sólo en el caso de una economía en equilibrio general se justificaría esa convicción de que podrán efectivamente comprar y vender tanto como ellos desearan. Pero sólo en el caso de una economía en equilibrio general se justificaría esa convicción ya que al no haber equilibrio entre ofertas y demandas en algún mercado, algunos agentes se verían restringidos en sus deseos de intercambio. A partir de la inseguridad de que la economía pueda permanecer siempre en equilibrio, ${ }^{13}$ es lógico pensar que los agentes expresen sus demandas y ofertas dadas las restricciones que perciben sobre las cantidades que se puedan intercambiar: sus demandas y ofertas se vuelven efectivas y se hace posible la existencia de un estado de equilibrio bajo restricciones, o equilibrio "keynesiano".

La reformulación keynesiana de un modelo de equilibrio general debería entonces incluir restricciones eventuales sobre cantidades intercambiables. Del lado del consumidor se expresaría como:

$$
\begin{aligned}
& \operatorname{Max} U\left(\bar{X}_{h}-x_{h}\right) \\
& x_{h} \\
& \text { dado que: } P x_{h} \geqslant 0 \\
& x_{h} \leqslant \bar{Y}_{h} \quad h=1,2, \ldots n
\end{aligned}
$$

en donde $\bar{Y}_{h}$ es un vector de cantidades máximas esperadas de intercambio por el agente $h$ en todos los mercados.

Del lado de las empresas, el problema equivalente sería:

$$
\begin{array}{ll}
\operatorname{Max} P Y_{f} & \\
Y_{f} \\
\text { dado que: } Y_{f} \in F_{f} \\
\\
Y_{f} \leqslant \bar{Y}_{f}
\end{array}
$$

13 Se vio antes que la posibilidad de existencia de un estado de desequilibrio era muy alta debido al considerable procesamiento de información que se requería para alcanzar el equilibrio. 
$\mathrm{Al}$ agregar todas estas ofertas y demandas, se puede entonces deducir un vector agregado de ofertas netas, función de los precios, de los acervos y de las cantidades máximas esperadas de intercambio:

$$
Z(P, \bar{Y}, \bar{x})=\sum_{h=1}^{n} x_{h}\left(P, \bar{Y}_{h}, \bar{x}_{h}\right)+\sum_{f=1}^{m} Y_{f}\left(P, \bar{Y}_{f}\right)
$$

En cuanto las restricciones sobre cantidades no sean activas (al ser mayores o iguales que las cantidades nocionales) estas ofertas serán idénticas a las ofertas walrasianas derivadas en una economía sin restricciones. Pero a partir del momento en que alguna restricción opere, las ofertas se vuelven efectivas y el equilibrio alcanzado ya no es walrasiano. ${ }^{14}$

Antes de aplicar esta formulación a los modelos agregados derivados antes es interesante notar que no se requiere apartarse de la hipótesis de competencia perfecta, hipótesis que Keynes implícitamente aceptó. Sin embargo, es claro que a partir de una formulación dentro de un marco de competencia monopolista se alcanzarían funciones de oferta y demanda con características similares, o sea en las cuales entrarían ciertos pará. metros de intensidad de demanda, parámetros a su vez funciones del nivel percibido de actividad en el intercambio. Una situación de competencia imperfecta por lo tanto se armoniza del todo con una formulación keynesiana aun cuando esta teoría haya sido originalmente concebida dentro de un marco de competencia perfecta. ${ }^{15}$

\section{Un modelo "pseudo-keynesiano" sencillo}

A partir del esquema del modelo neoclásico expuesto antes, a nivel agregado se puede derivar un modelo de tipo keynesiano con tres mercados (trabajo, bienes y dinero) y tres precios (el precio de los bienes, el sueldo y la tasa de interés o el precio de los bienes futuros). El único acervo considerado explícitamente es la masa monetaria real y los demás acervos se consideran fijos dentro del horizonte de corto plazo del modelo. El vector de restricciones sobre cantidades se reduce a un solo componente: el producto total de la economía, ligado al nivel global de empleo a través de una función agregada de producción; las restricciones percibidas sobre el empleo y sobre las ventas varían entonces en forma paralela. Por último, se establece la hipótesis de que las expectativas sobre cantidades intercambiables se ajustan rápidamente a un estado de equilibrio de corto plazo, de tal forma que las expectativas de producción corresponden al nivel de producción de equilibrio: $\bar{Y}=Y$.

\footnotetext{
14 Para una discusión y derivaciones de equilibrio no walrasiano dentro de un marco conceptual general, véase en particular Dreze (1973), Benassy (1974), Grandmont y Laroque (1976) y Malinvaud (1977).

15 Sobre este punto véase Bennassy (1976).
} 

como:

El equilibrio en el mercado de trabajo se puede entonces expresar

$$
L^{o}\left(\frac{w}{P}, r, \frac{M}{P}\right)=L^{d}\left(\frac{w}{P}, r, \frac{M}{P}, Y\right)
$$

El equilibrio en el mercado de bienes:

$$
Y=C\left(\frac{w}{P}, r, \frac{M}{P}, Y\right)+I\left(\frac{w}{P}, r, \frac{M}{P}, Y\right)
$$

Por último, en el mercado de dinero:

$$
M^{o}=M^{a}\left(\frac{w}{P}, r, \frac{M}{P}, Y\right)
$$

En este modelo hay cuatro variables $(w, P, r, Y)$ y sólo tres ecuaciones. Falta una ecuación y es fácil ver por qué: cuando se altera el estado de equilibrio, ese cambio se puede traducir principalmente ya sea en un ajuste en cantidades $(Y)$ o en un ajuste en salarios y/o precios $(w, P)$ : No hay nada en el modelo que a priori permita predecir cómo se va a realizar el ajuste. La determinación simúltanea de precios y de cantidades por empresas y consumidores es una de las principales piezas faltantes de la teoría keynesiana. Más adelante se analizan algunas de las razones por las cuales este eslabón no ha sido integrado aún.

\section{El modelo neokeynesiano usual}

Keynes sugiere en realidad que salarios y precios tienden a ser rígidos a la baja, o sea que existen restricciones de corto plazo sobre precios y salarios:

$$
w \geqslant \vec{w}, p \geqslant \vec{p}
$$

Cuando ambas restricciones son activas, sobra un grado de libertad en el modelo, la condición que Keynes elimina es la de equilibrio en el mercado de trabajo, o sea que abre la posibilidad de un estado de desempleo duradero.

Sin embargo, esa hipótesis no elimina el grado de libertad que sigue existiendo cuando ni salarios ni precios se encuentran restringidos. Los neokeynesianos resuelven este problema al suponer que salarios y precios son totalmente rígidos cuando la economía se encuentra en el interior de su frontera productiva (o sea que existe desempleo) y se ajustan inmediatamente en alza cuando se empuja la economía contra su frontera, o sea que:

$$
w=\bar{w}, P=\bar{P} \text { cuando } Y<Y_{f}
$$


donde $Y_{f}$ es el nivel de producción de pleno empleo. Sin embargo, esta hipótesis ha sido severamente criticada en los últimos años como contraria al pensamiento original de Keynes y como inadecuada en su representación de la realidad. Regresaremos después sobre este punto.

Por otra parte, es preciso notar que en el modelo keynesiano el dinero no tiene razón de ser neutral: al ser $w$ y $P$ dados y rígidos, una variación en la oferta monetaria por necesidad va a influir paramétricamente sobre las funciones de oferta y demanda con lo cual se altera el nivel de actividad económica y la tasa de interés. No existe ya entonces una dicotomía entre el lado real y el lado monetario sino que el estado de la economía es una resultante de factores a la vez reales y monetarios.

\section{Interdependencia y fallas de coordinación}

La inclusión de expectativas sobre cantidades intercambiables en las funciones de oferta y demanda introduce una interdependencia directa entre los comportamientos de los agentes: el comportamiento del agente $A$ se vuelve una función de sus expectativas sobre el comportamiento del agente $B$ el cual a su vez depende de sus expectativas sobre el comportamiento de $A$, etc... La independencia ideal del modelo walrasiano, en el cual no hay más interacción que a través de un vector de precios, se desvanece y se establece una situación de juego entre todos los participantes. Se puede dar entonces un estado de equilibrio ineficiente en el sentido de Pareto: todos los agentes pueden tener interés en ponerse de acuerdo para alterar de manera simultánea su comportamiento, pero no existe un mecanismo que lo permita; existen "fallas de coordinación" en la terminología de Leijonhufvud.

Un ejemplo bien conocido de falta de coordinación en el ámbito de la literatura sociológica es el caso del dilema del prisionero. Dos prisioneros son interrogados por separado (sin poder consultarse previamente) por un crimen que supuestamente han cometido juntos. Si ambas se niegan a reconocer su culpabilidad se les tendrá que liberar; pero si uno la niega mientras el otro la reconoce, el que niega es condenado a muerte. Si ambos aceptan su culpabilidad, se les da a ambos una pena importante de cárcel. Si un prisionero teme que el otro confiese probablemente se decida por confesar. Si ambos confiesan obtienen una pena importante, pero hubieran podido salir libres de no confesar. El problema de fondo es una falta de coordinación entre participantes.

Si se realiza el mismo tipo de juego repetidas veces, las expectativas de los participantes tenderán a depender del comportamiento de los demás jugadores en el pasado. En el caso de los prisioneros, si siempre han confesado en el pasado, seguirán confesando en el futuro: el sistema permanece en un estado de equilibrio ineficiente.

El equilibrio keynesiano de depresión es un ejemplo tipo de falta de coordinación: si empresas y trabajadores se pudieran poner de acuerdo 
para incrementar sus compras de manera simultánea (de bienes por los consumidores y de trabajo por las empresas) la economía saldría de la depresión, alcanzándose así un estado más eficiente. Sin embargo, no existe un mecanismo que lo permita; si un agente actuara en forma aislada saldría perjudicado y por lo tanto no lo hace. ${ }^{16}$

\section{HACIA UNA REFORMULACIÓN DEL MODELO KEYNESIANO}

\section{Hacia una mayor desagregación}

El primer paso indispensable para extraer a la teoría keynesiana fuera del marco rígido y sesgante del modelo neokeynesiano es de regresar hacia una visión más desagregada de la economía, más cerca a la vez del marco desagregado de la teoría del equilibrio general y del pensamiento keynesiano original.

Con toda probabilidad, uno de los puntos más importantes es pasar del modelo de un sector con un solo bien homogéneo a un modelo de por lo menos dos sectores, diferenciando así bienes de consumo y de inversión. No se detalla este punto pero basta con revisar la literatura correspondiente para apreciar que un modelo de dos sectores permite ampliar grandemente el realismo y la riqueza del modelo neokevnesiano. ${ }^{17}$

Otra área de necesaria desagregación es la de la distribución de los acervos entre los agentes, en particular de los acervos monetarios y financieros. La distribución del dinero y de los activos y pasivos financieros en un contexto de riesgo e incertidumbre puede hacer variar de manera radical los flujos agregados de oferta y demanda al imponer limitaciones brutales en la capacidad de pago de los agentes, con posibilidades de bancarrota en cadena. Estas cuestiones han empezado a ser explotadas de manera sistemática; ${ }^{18}$ conducen naturalmente hacia una integración dentro del modelo keynesiano de aspectos de intermediación financiera dentro de un contexto de riesgo e incertidumbre.

Una tercera área de desagregación corcierne a los tipos de activos financieros cuyo precio varía al ser fijado por el juego de la demanda y de

16 Existen muchos otros ejemplos de equilibrios económicos ineficientes que se deben a la interdependencia de las expectativas de comportamiento. Uno de ellos es el de la inflación permanente causada por revisiones de precios y salarios: si cada agente piensa que los demás agentes van a incrementar sus precios de oferta decidirá hacer lo mismo, perpetuándose así el estado de inflación.

17 Véase por ejemplo el modelo de dos sectores de Foley y Sidrauski (1971). Es interesante señalar que existe cierta polémica acerca del pensamiento original de Keynes en ese sentido, Leijonhufvud (1968) argumenta que Keynes formula su modelo de la Teoria general en términos de dos sectores productivos pero otros han sostenido lo opuesto.

18 Véase por ejemplo Hahn (1965), Minsky (1976), Foley y Córdoba (1971), Aumann y Kurz (1977). 
la oferta en una bolsa de valores. Es un punto sobre el cual Keynes insistió en su Teoría general y que algunos autores ${ }^{19}$ han catalogado como ingrediente esencial de las crisis y fluctuaciones económicas de mediano plazo.

\section{Hacia una formulación dinámica}

E1 segundo paso necesario para rescatar al modelo keynesiano consiste en restituirle su dimensión dinámica. ${ }^{20}$ Para eso es preciso colocar las expectativas sobre condiciones futuras de intercambio (tanto precios como cantidades que se esperan poder intercambiar a estos precios) al centro del análisis, en vez de dejarlas en la oscuridad como sucede en el modelo neokeynesiano.

Las decisiones de intercambio se consideran hoy en función de las expectativas sobre condiciones futuras de intercambio: esto se debe a que existen acervos (como capital físico, capital humano, capital financiero, inventarios, etc....) que, al sobrevivir de un período a otro, permiten realizar transferencias intertemporales de flujos.

Un consumidor puede por ejemplo decidir consumir más ahora y menos mañana por medio de un incremento en su nivel de deuda. Una empresa puede producir más ahora para vender más en el futuro mediante un incremento de sus inventarios de productos terminados, etc... Probablemente lo más realista en una economía con información imperfecta es pensar que las expectaciones se forman con base en un proceso de aprendizaje sobre las condiciones pasadas de intercambio. Un cambio en las condiciones actuales de intercambio debería entonces alterar en cierta medida el estado de expectativas, ${ }^{21}$ lo cual a su vez tendería a modificar el deseo de los agentes por poseer acervos. Un cambio en el deseo de poseer acervos debería entonces repercutir en los flujos agregados de ofertas y demanda y por lo tanto en el estado actual de intercambio, y se cerraría así el proceso dinámica de retroalimentación representado en la gráfica 1 .

Dentro de ese proceso intertemporal habría también que incluir a los cambios habidos en la distribución de acervos entre los agentes, cambios que pueden imprimir una dinámica propia al sistema al modificar las funciones agregadas de oferta y demanda.

Una formulación keynesiana completa debería entonces seguir este

19 Véase por ejemplo Minsky (1976).

20 Una de las ventajas de una formulación dinámica, es que permite eliminar la distinción artificial entre corto y largo plazo, modelos de fluctuación del ingreso y modelos de crecimiento.

21 El estado de expectativas incluye expectativas de precios a muy corto plazo; cambios en esas expectativas pueden modificar el valor percibido de los acervos en posesión de los agentes: por ejemplo, precios de acciones en la bolsa de valores. 


\section{Gráfica 1}

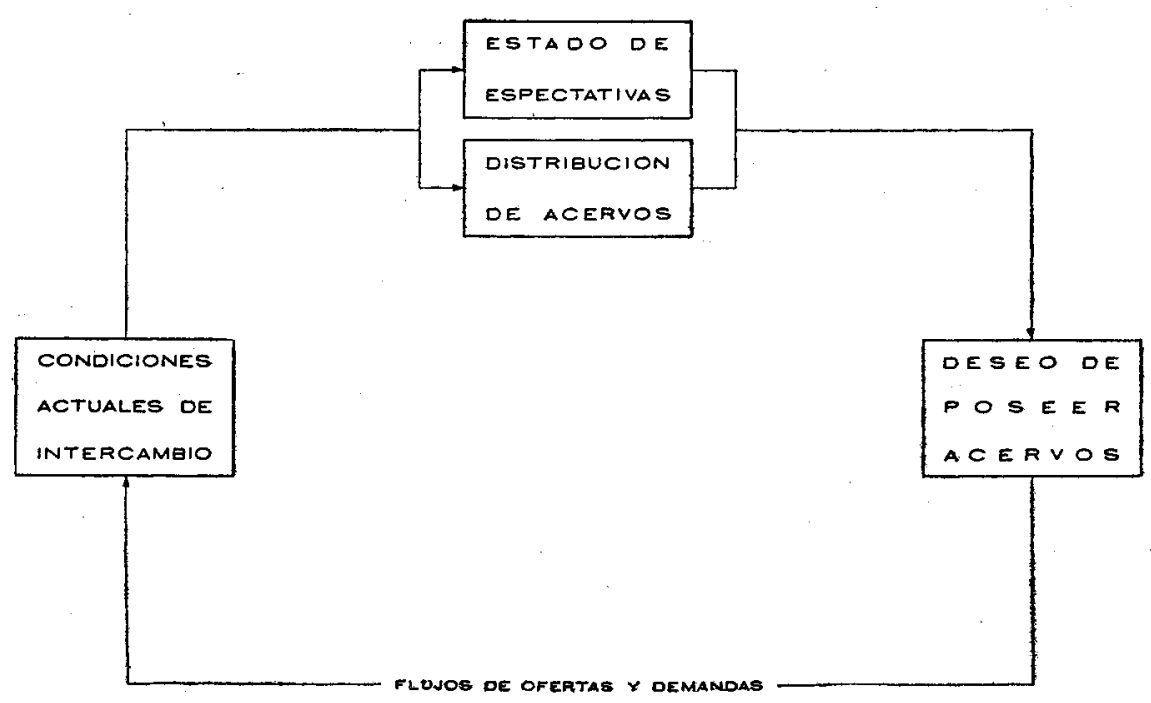

esquema dinámico. ${ }^{22}$ Sin embargo, el problema radica en la definición adecuada de cada uno de estos eslabones, en particular del proceso de formación de expectativas y del deseo de poseer acervos: mientras la formación de expectativas depende de factores sociológicos, sicológicos e institucionales diversos, el deseo de poseer acervos es una resultante compleja de la estructura productiva, de las preferencias intertemporales, de las aversiones al riesgo y de la naturaleza y variedad de los instrumentos financieros existentes. Mucho queda por hacer en estas áreas. ${ }^{23}$

\section{Hacia una teoría de la formación de precios y de la tasa de ganancia}

Se mencionó antes que la formulación keynesiana al abandonar la hipótesis de equilibrio permanente en el mercado de trabajo, $\mathrm{y}$ al introducir el ingreso como parámetro adicional de las funciones de oferta y demanda, tendría dos grados de libertad en el modelo, o sea que ni el nivel de precios ni la distribución entre salarios y precios (o salario real) estaban en general definidos. Es necesario integrar entonces dentro del marco keynesiano tanto una teoría de la formación de los precios como de la distribución entre salarios y precios, o sea de la tasa de ganancia.

22 Un modelo que parte de bases microeconómicas totalmente definidas y sigue los lineamientos generales especificados aquí, fue desarrollado por lze (1977a).

23 Algunos desarrollos recientes en el ambiente del comportamiento dinámico de una economía keynesiana se encuentran en Leijonhufvud (1973), Minsky (1976), Homitt (1977) e Ize (1977b). 
En una economía perfectamente competitiva y sin restricciones de intercambio, la tasa de ganancia se obtendría como producto de un estado de equilibrio general que permita igualar el salario real con el producto marginal del trabajo, o sea que sería fijada por una interacción "mecánica" de todos los agentes a través de un conjunto de mercados. Sin embargo, en una economía en equilibrio keynesiano con restricciones de intercambio ya no hay igualdad entre el salario real y el producto marginal y esto explica que la tasa de ganancia permanezca indefinida. ${ }^{24}$ El problema de fondo es entonces el siguiente: ya que el mecanismo de mercado, aun en un contexto de competencia perfecta, no permite fijar los precios de oferta (tanto salarios como precios), es inevitable que sean los agentes ellos mismos quienes los fijen, o sea que existe de hecho un estado de competencia monopolista. ¿Cómo fijan entonces las empresas y los trabajadores sus precios de oferta en ese contexto?

Un primer elemento de respuesta concierne a la velocidad de ajuste de los precios en un contexto de información imperfecta: cada agente tiene información parcial sobre lo que está pasando en la economía y puede percibir información adicional por medio de un proceso de búsqueda; por otra parte, se enfrenta con ciertos costos de transacción al alterar sus precios. Al juntar ambos elementos se pueden entonces deducir ciertas características de los ajustes de precios, en particular de rigideces temporales a la baja y de ajustes relativamente rápidos a la alza, características que fundamentan bastante bien la visión keynesiana ${ }^{25}$

Estos nuevos desarrollos, basados en problemas de información, permiten explicar ciertas dinámicas de ajustes de precios; sin embargo, no explican cómo se fija su nivel absoluto. Esta última pregunta no se puede contestar sin introducir elementos de economía política dentro de un proceso complejo de dinámica social que incluye conflictos entre clases de agentes, coaliciones, poder político y que asigna un papel dominante a la formación de expectativas. Esto nos ubica fuera del ámbito de la competencia perfecta, pero dentro de un contexto de interacciones oligopólicas y de cierta dinámica histórica conjunta de evolución de las condiciones sociales y de producción. La falla de la teoría keynesiana en explicar situaciones de desempleo con inflación como las registradas en el último decenio es probablemente una de las consecuencias del olvido de estos elementos.

Esta teoría, al reconocer las interdependencias de los comportamientos que se establecen a través de las expectativas de intercambio, ha logrado explicar la existencia de equilibrios depresivos y cierta dinámica

24 Sin embargo, es preciso notar que Keynes mantiene como válida la hipótesis de igualdad del salario real y del producto marginal del trabajo sin considerar que esa hipótesis es inconsistente con una situación en la cual las empresas no pueden vender tanto como quieran.

$25 \mathrm{Ya}$ existe una extensa literatura sobre la formación de precios en un contexto de información imperfecta. Véanse en particular los trabajos reunidos en Phelps (1970) y la reseña de Rostchild (1973). 
cíclica alrededor de esos equilibrios; se ha dado así un primer paso, decisivo, para alejarse del mecanismo de la teoría clásica. El segundo paso consistiría ahora en reconocer las interdependencias sociales de los agentes, acercándose así por ejemplo a teorías de economía política como la teoría marxista. Solo en esta forma probablemente se podrá explicar la dinámica conjunta de precios y cantidades.

\section{El EQUILIBRIO GENERAL COMPETITIVO EN RETROSPECTIVA}

Concluiremos este ensayo con una nota breve: si el gran logro de la economía keynesiana fue alejarse del mecanismo del modelo clásico y de su pariente conceptual, el equilibrio general competitivo, y si es necesario para que se vuelva más realista que se aleje aún más, ¿por qué no abandonar en las tinieblas del olvido a la teoría del equilibrio general? En otras palabras, ¿en qué nos ha sido útil esa teoría como punto de partida de esta discusión?

El interés principal del equilibrio general competitivo es que ofrece un marco de referencia conceptual de gran utilidad para iluminar, a veces de manera directa y a veces por contraste, a un conjunto de mecanismos y problemas. El enfoque microeconómico y la forma de concebir las interacciones de un conjunto de agentes a través de un conjunto de mercados intertemporales es sin duda una visión muy rica y profunda. Las hipótesis particulares de competencia perfecta, demandas nocionales, información perfecta, convexidades, etc., pueden ser abandonadas por uno, pero el marco conceptual puede permanecer. Aun si se conservan esas hipótesis el modelo es útil para esclarecer varios mecanismos y problemas de una economía real: demandas efectivas, problemas de información, fallas sociales, etc. El mismo concepto de equilibrio general es indispensable para ubicar mejor conceptos derivados como el del equilibrio keynesiano bajo restricciones, o simplemente una dinámica de desequilibrio: como la misma palabra lo señala, un desequilibrio es ante todo un estado de neoequilibrio. Por último ciertos conceptos como el de las preferencias de los consumidores, de los conjuntos tecnológicos de las empresas y de la maximización individual, son elementos útiles para casi cualquier teoría económica: la teoría del equilibrio general ofrece un ejemplo particularmente sencillo pero irrealista de uso de estos conceptos; nada impide modificarlos y ensamblarlos dentro de un marco a la vez más complejo y menos utópico.

Es interesante y algo paradójico concluir entonces que si el gran logro de Keynes fue de apartarse de la teoría clásica, el gran acierto en los últimos dos decenios fue de rescatar a Keynes del modelo keynesiano mediante un reacercamiento con el espíritu de la teoría del equilibrio general. 


\section{BIBLIOGRAFIA}

Arrow, K. J. y F. H. Hahn (1971), General Competitive Analysis, San Francisco, Holden-Day.

Aumann, R. J. y M. Kurz (1976), "Incomplete Markets and Bankruptcy: A Walrasian Perspective", IMsss Technical Reports.

Barro, R. J. y H. I. Grossman (1971), "A General Disequilibrium Model of Income and Employment", American Economic Review.

Benassy, J. P. (1974), "Neo-Keynesian Disequilibrium in a Monetary Economy", Review of Economic Studies.

Benassy, J. P. (1976), "The Disequilibrium Approach to Monopolistic Price Setting and General Monopolistic Equilibrium", Review of Economic Studies.

Clower, R. W. (1965), "The Keynesian Counterrevolution: A Theoretical Appraisal" en F. H. Hahn y F. P. R. Brechling (Comps.), The Theory of Interest Rates, Londres, Macmillan.

Dreze, J. (1973), "Existence of an Equilibrium under Price Rigidities", coRE Discussion Paper.

Foley, D. y I. Córdoba (1976), "A Model of the Circulation of Money", Documento de trabajo, Stanford University.

Foley, D. y M. Sidrauski (1971), Monetary and Fiscal Policy in a Growing Economy, Londres, Macmillan.

Grandmont, J. M. (1976), "A Temporary General Equilibrium Theory", CEPREMAP.

Grandmont, J. M. y G. Laroque (1976), "On Temporary Keynesian Equilibria", Review of Economic Studies.

Grandmont, J. M. y Y. Younés (1973), "On the Role of Money and the Existence of a Monetary Equilibrium", Review of Economic Studies.

Green, J. R. (1973), "Temporary General Equilibrium in a Sequential Trading Economy with Spot and Future Transactions", Econometrica.

Hahn, F. H. (1965), "On Some Problems of Proving the Existence of Equilibrium in a Monetary Economy" en F. H. Hahn y F. P. R. Brechling (Comps.), The Theory of Interest Rates, Londres, Macmillan.

Heller, W. P. y R. M. Starr (1976), "Unemployment Equilibrium with Rational Expectations", IMsss Technical Reports.

Howitt, P. (1977), "The Limits to Stability of a Full-Employment Equilibrium", Documento de Trabajo, The University of Western Ontario.

Ize, A. (1977a), "Corridor Effects in a Monetary Economy", Documento de Trabajo, Stanford University.

Ize, A. (1977b), "Disequilibrium Dynamics and Corridor Effects in a Keynesian System", Documento de Trabajo, El Colegio de México.

Leijonhufvud, A. (1968), On Keynesian Economics and the Economics of Keynes, Nueva York, Oxford University Press.

Leijonhufvud, A. (1973), "Effective Demand Failures", Swedish Journal of Economics.

Leijonhufvud, A. (1974), "The Varieties of Price Theory: what Microfoundations for Macro Theory?' Documento de Trabajo, UcLA. 
Malinvaud, E. (1977), The Theory of Unemployment Reconsidered, Nueva York, John Wiley.

Minsky, H. P. (1975), John Maynard Keynes, Nueva York, Columbia University Press.

Patinkin, D. (1956), Money, Interest and Prices, Nueva York, Harper and Row.

Phelps, E. S. et al. (1970), Microeconomic Foundations of Employment and Inflation Theory, Nueva York, Norton.

Radner, R. (1972) "Existence of Equilibrium Plans, Prices and Prices Expectations in a Sequence of Markets", Econometrica.

Rostchild, M. (1973), "Models of Market Organization with Imperfect Information", Journal of Political Economy.

Weintraub, R. (1977), "The Microfoundations of Macroeconomics: A Survey", Journal of Economic Literature. 